\title{
The emerging association of canonical finger patterns and quantity- number linkage in early childhood
}

\author{
Julia Bahnmueller ${ }^{1,4^{*}}$, Roberta Barrocas ${ }^{2}$, Korbinian Moeller ${ }^{1,2,4,5}$, Stephanie Roesch ${ }^{3,4}$ \\ ${ }^{1}$ Centre for Mathematical Cognition, Loughborough University, University Road, LE11 3TU, \\ Loughborough, United Kingdom \\ ${ }^{2}$ Leibniz-Institut für Wissensmedien, Schleichstraße 6, 72076 Tübingen, Germany \\ ${ }^{3}$ Hector Research Institute of Education Sciences and Psychology, University of Tübingen, \\ Europastraße 6, 7072 Tübingen, Germany \\ ${ }^{4}$ LEAD Graduate School \& Research Network, University of Tübingen, Europastraße 6, 7072 \\ Tübingen, Germany \\ ${ }^{5}$ IDeA Centre, Rostocker Straße 6, 60323 Frankfurt am Main, Germany
}

\begin{abstract}
Author note
Julia Bahnmueller: Correspondence concerning this article should be addressed to Julia Bahnmueller, Centre for Mathematical Cognition, Loughborough University, Schofield building (SCH.0.30), University Road, Loughborough, LE11 3TU, United Kingdom, email: j.bahnmuller@1boro.ac.uk. Roberta Barrocas: Leibniz-Institut für Wissensmedien, Schleichstraße 6, 72076 Tübingen, Germany, email: r.barrocas@iwm-tuebingen.de. Korbinian Moeller: Centre for Mathematical Cognition, Loughborough University, University Road, Loughborough, LE11 3TU, United Kingdom, email: k.moeller@lboro.ac.uk; Stephanie Roesch: Hector Research Institute of Education Sciences and Psychology, University of Tübingen, Europastraße 6, 7072 Tübingen, Germany, email: stephanie.roesch@unituebingen.de.
\end{abstract}




\begin{abstract}
Through repeated use of fingers for counting and representing numerical magnitudes in early childhood, specific finger patterns become associated with mental representations of specific quantities. Although children as young as three years of age already use their fingers for representing numerical quantities, evidence on advantageous recognition of such canonical compared to non-canonical finger patterns as well as its association with numerical skills in young children is scarce. In this study, we investigated the performance of $N=101$ children aged around four years in canonical vs. non-canonical finger pattern recognition and its concurrent association with skills tapping into children's' knowledge about quantity-number linkage. Extending previous findings observed for older children, the present results indicated that despite considerable variability on the individual level performance in canonical finger pattern recognition was better compared to non-canonical finger pattern recognition on the group level. Moreover, both canonical and non-canonical finger pattern recognition was positively correlated with tasks tapping into quantity-number linkage. However, when controlling for verbal counting skills, correlations that remained significant were only found for canonical but not non-canonical finger pattern recognition performance. Overall, these results provide insights into the early onset and significance of the effect of canonicity in finger pattern recognition during early numerical development.
\end{abstract}

(Word count: 202)

Key words: finger-pattern recognition, canonicity advantage, finger-based representation, finger-based strategy, numerical development, counting

PsychINFO classification codes: 2820 Cognitive \& Perceptual Development, 2340 Cognitive Processes, 2343 Learning \& Memory, 3550 Academic Learning \& Achievement 
FINGER PATTERNS IN EARLY CHILDHOOD

Children across very different cultures are exposed to and often use their fingers for representing, manipulating, and acquiring numerical information (e.g., Butterworth, 1999). Recent evidence suggests that finger-based numerical strategies (i.e., finger counting and use of cardinal finger patterns) play an important, potentially functional role for learning skills related to counting (e.g., Crollen \& Noël, 2015; Crollen et al., 2011) and quantity-number linkage (e.g., Gibson et al., 2019). One suggested mechanism by which finger-based strategies might aid the development of numerical skills seems to lay in the formation of iconic, embodied finger-based representations of number magnitude (cf. Beller \& Bender, 2011; Di Luca \& Pesenti, 2011; Moeller et al., 2012): Through repeated use of finger-based numerical strategies in early childhood, specific - so-called canonical - finger patterns matching individual and cultural finger counting habits seem to become associated with the respective numerical magnitude they represent (e.g., thumb and index finger for two in the usual German counting habit). This is reflected in a processing advantage for canonical over non-canonical finger patterns previously observed in children aged five years and older (Kreilinger et al., 2021; Lafay et al., 2013; Marlair et al., 2021; Noël, 2005) but also adults (e.g., Barrocas et al., 2020; Di Luca \& Pesenti, 2008; Soylu et al., 2019; Van den Berg et al., 2021).

Accordingly, it has been suggested that using fingers for representing and manipulating numerical information may result in iconic, finger-based representations of number magnitude in the form of canonical finger patterns (Di Luca \& Pesenti, 2011). Crucially, in contrast to other representations of number (i.e., number words and Arabic digits), finger-based representations also represent a specific case of embodied cognition (cf. Barsalou, 2008, Lakoff \& Núñez, 2000) as they are rooted in and allow for continued sensory-motor experiences of early finger counting and calculation. As such, iconic, embodied finger-based representations of number magnitude may represent the "missing 
FINGER PATTERNS IN EARLY CHILDHOOD

link” (Fayol \& Seron, 2005, see also Butterworth, 1999) for relating symbolic number representations (i.e., number words and Arabic digits) with non-symbolic quantities and thus for facilitating the transition from concrete to abstract representations of number (cf. Andres et al., 2008). Against this background, the current study set out to broaden our understanding of the developmental onset of the emergence of such iconic, embodied finger-based representations and their association with concurrent numerical skills (i.e., quantity-number linkage) in young children around the age of four years.

Several studies suggested (canonical) finger pattern recognition performance to be associated with concurrent and predictive of future numerical but also arithmetic skills (e.g., Cornu et al., 2018; Hornung et al., 2017; Kreilinger et al., 2021; van Rinsveld et al., 2020). For instance, Cornu and colleagues (2018) showed that canonical finger pattern recognition performance was associated with concurrent counting and number naming skills in a sample of five-to-six-old children at the end of kindergarten. Two additional studies by Kreilinger et al. (2021) and Hornung et al. (2017) further indicated that canonical finger pattern recognition assessed at the end of the last year of kindergarten (at about six years of age) and at the beginning of first grade (at about six-to-seven years old), respectively, predicted first graders' arithmetic performance longitudinally (i.e., assessed four and eight months later in first grade, respectively). Moreover, canonical finger pattern recognition performance in fiveto-six-year-old children in their last year of kindergarten was found to predict their performance in a number line estimation task about nine months later at the beginning of first grade, even when controlling for the influence of basic counting skills (van Rinsveld et al., 2020).

However, studies focusing exclusively on the relation of the recognition of canonical finger patterns and numerical/arithmetic skills do not readily allow for strong claims about 


\section{FINGER PATTERNS IN EARLY CHILDHOOD}

the underlying mechanisms driving the observed associations. While (automatic) access to canonical finger-based representations of number magnitude might be one plausible explanation, efficient counting but also subitising strategies (i.e., recognising a small set of objects without counting, e.g., Kaufmann et al., 1949) may account for the observed relations between finger pattern recognition and numerical or arithmetical skills as well. In this context, considering unique effects of canonical vs. non-canonical finger pattern recognition might help specify the precise effect of canonical finger-based representations from the use of counting and subitising strategies.

Contrasting performance in canonical vs. non-canonical finger pattern recognition, previous studies showed a consistent canonicity advantage; this means, better performance in recognising canonical compared to non-canonical finger patterns in both adults (e.g., Barrocas et al., 2020; Di Luca \& Pesenti, 2008; Soylu et al., 2019; Van den Berg et al., 2021) and children around five years and older (Kreilinger et al., 2021; Lafay et al., 2013; Marlair et al., 2021; Noël, 2005). To better understand the working mechanisms underlying this processing advantage for canonical finger patterns, Di Luca and colleagues conducted a series of experiments indicating that adults automatically activated number semantics (i.e., numerical magnitude) for canonical but not for non-canonical finger patterns (Di Luca \& Pesenti, 2008). Additionally, their results showed that canonical finger patterns seem to be processed similarly to other number symbols (e.g., Arabic digits), whereas processing of noncanonical finger patterns rather resembled processing of non-symbolic quantities (Di Luca et al., 2010; for converging ERP evidence see Soylu et al., 2019, and Van den Berg et al., 2021). Taken together, these studies provide convincing support for the idea of embodied finger-based representations of (small) numbers in adults suggesting a direct link and automatic access to magnitude information through iconic, canonical finger patterns. 
FINGER PATTERNS IN EARLY CHILDHOOD

Comparable systematic experimental work is lacking across numerical development. Nevertheless, several studies provided evidence for the existence and significance of differential performance in canonical compared to non-canonical finger pattern recognition in kindergarten and elementary school children. Overall, previous studies consistently indicated a canonicity advantage for children above the age of five (Lafay et al., 2013; Marlair et al., 2021; Noël, 2005). Although children as young as three years old already use their fingers for representing numerical quantities (e.g., Gunderson et al., 2015), to the best of our knowledge, only one study investigated the effect of canonicity in children below the age of five. In their cross-sectional study, Lafay and colleagues (2013) tested three relatively small groups of children (about 20 children per group): preschool children (aged four-to-five years), kindergarten children (aged five-to-six years), and children in grade 1 (aged six-to-seven years) on both a finger pattern comparison and a finger pattern recognition task. Children of all three groups were better able to indicate whether two finger patterns showed the same quantity when they were both presented as canonical patterns as compared to when they were presented as non-canonical or mixed finger patterns. However, while a canonicity advantage was observed for the two older age groups in the finger pattern recognition task, no significant processing advantage was observed for canonical as compared to non-canonical finger patterns for the youngest group of children. Crucially, the youngest age group performed worse compared to the two other groups in both canonical and non-canonical pattern recognition suggesting that children aged five and younger may not yet have established sufficiently solid finger-based representations of number magnitude for canonical finger patterns but may rather still use a combination of counting and recognition strategies (or solely rely on counting).

This interpretation is further corroborated by evidence suggesting that the size of the canonicity advantage seems to depend on both the quantity range covered and the age of 
FINGER PATTERNS IN EARLY CHILDHOOD

children tested (Kreilinger et al., 2021; Noël, 2005; Marlair et al., 2021). For instance, Noël (2005) reported a more pronounced canonicity advantage for larger (six to nine) compared to smaller quantities (two to five) in second graders aged about seven years. Kreilinger and colleagues (2021) who compared performance in canonical finger pattern recognition with performance in enumerating small unstructured sets observed a processing advantage for canonical finger patterns for quantities five and six but not quantities one to four in six-yearolds at the end of kindergarten. Moreover, investigating a sample of fourth and fifth graders around the age of ten, results of Marlair and colleagues (2021) suggested an inverse u-shaped pattern for finger patterns from four to nine. In particular, in this study the canonicity advantage was shown to be smallest for quantities four and nine with a peak at quantities six and seven. Taken together, the results of these studies suggest that for children aged six years and older the canonicity advantage is more pronounced for larger quantities (i.e., larger than four). However, data is lacking for younger children for whom an (emerging) canonicity advantage might (still) be evident for a smaller quantity range.

Several studies demonstrated that canonical finger recognition performance is associated with other basic numerical skills (e.g., counting and number naming: Cornu et al., 2018; arithmetic: Hornung et al., 2017; Kreilinger et al., 2021; number line estimation: van Rinsveld et al., 2020). However, there is a clear research gap regarding studies specifically investigating differential associations of canonical and non-canonical finger pattern recognition with numerical skills in young children. First hints pointing towards a benefit through the advantageous processing of canonical finger patterns for numerical development may be drawn from above mentioned study by Kreilinger and colleagues (2021) who compared performance in canonical finger pattern recognition with performance in enumerating small unstructured sets. Results of this study indicated that finger pattern recognition but not enumeration of unstructured sets measured in six-year-olds at the end of 
FINGER PATTERNS IN EARLY CHILDHOOD

kindergarten were predictive of addition performance in first grade. Another study by Barrocas and colleagues (2020) further showed differential associations of canonical vs. noncanonical finger pattern recognition with the four basic arithmetic operations in adults.

Thus, while there is initial evidence for differential associations of canonical vs. noncanonical finger pattern recognition with arithmetic performance (in adults), studies addressing differential associations with effects on more basic numerical skills are still lacking. However, in case finger-based representations of number magnitude (as indexed by the advantageous recognition of canonical over non-canonical finger patterns) indeed facilitate linking symbolic number representations (i.e., number words and Arabic digits) with non-symbolic quantities in early numerical development (cf. Fayol \& Seron, 2005), one would expect that children who show a processing advantage for canonical over noncanonical finger patterns should also perform better in tasks tapping into quantity-number linkage.

\section{The present study}

The aim of the present study was to broaden our understanding of the developmental onset of finger pattern recognition and its relation to concurrent numerical skills in young children around the age of four. To do so, we assessed performance of young children in canonical vs. non-canonical finger pattern recognition and their association with counting skills as well as quantity-number linkage.

Previous research supports the idea of a canonicity advantage (i.e., better recognition performance for canonical vs. non-canonical finger patterns) for children as young as five years and older (Lafay et al., 2013; Marlair et al., 2021; Noël, 2005) as well as for adults (Barrocas et al., 2020; Di Luca \& Pesenti, 2008; Soylu et al., 2019; Van den Berg et al., 2021). However, research regarding even younger children is scarce and did not substantiate 
FINGER PATTERNS IN EARLY CHILDHOOD

the existence of the canonicity advantage in younger children (Lafay et al., 2013). In the present study, we addressed two possible reasons which might qualify the canonicity advantage for canonical finger patterns in children below the age of five.

First, on the group level, the size of the canonicity effect is likely to be smaller for younger compared to older children due to substantial individual variation in the emergence of the effect (cf. Lafay et al., 2013). This might be driven by the fact that younger children may be more likely to use a mix of counting and recognition strategies when asked to name the number that corresponds to a presented finger pattern (Lafay et al., 2013). Second, counting and recognition strategies may further depend on the quantity represented by the finger pattern (i.e., the canonicity advantage might increase with the quantity represented by the finger pattern; cf. Noël, 2005). To account for aspects that may influence whether a canonicity advantage may be observed in children younger than five years of age, the present study was sufficiently powered to detect a small to medium sized effect of canonicity. Additionally, we specifically considered counting vs. recognition strategies for each of the quantities presented by finger patterns. Based on these considerations we expected that, while an effect of canonicity may not be detectable when considering all trials (solved by counting and recognition of finger patterns, cf. Lafay et al., 2013), a significant canonicity advantage should be observable when only considering those trials for which children did not openly show counting strategies. Furthermore, we expected the canonicity advantage to increase with the quantity represented by the finger pattern.

Following up on a limited number of studies investigating associations of (canonical and non-canonical) finger pattern recognition and concurrent and/or future numerical and arithmetic performance in children (Cornu et al., 2018; Hornung et al., 2017; Kreilinger et al., 2021; van Rinsveld et al., 2020), we further explored whether canonical and non-canonical 
FINGER PATTERNS IN EARLY CHILDHOOD

finger pattern recognition was (differentially) associated with counting skills as well as quantity-number linkage. Extending previous results by Cornu and colleagues (2018) indicating that canonical finger pattern recognition performance was associated with concurrent counting skills in a sample of five-to-six-year-old children, we expected this association to generalise to children aged around four years. Moreover, we assumed canonical finger pattern recognition to also be associated with performance in tasks tapping into quantity-number linkage (i.e., quantity-number mapping, Give-N, verbal number comparison).

\section{Methods}

\section{Sample}

A total of $1013-5$-year-old children were considered for the analyses reported in this article $(M=52.2$ months, $S D=5.0$, range $40-66$ months; $48 \%$ female, $52 \%$ male; $82 \%$ righthanded, $12 \%$ left-handed, $6 \%$ missing information on handedness). Of the initial sample of 122 children, 16 children for which at least one outcome measure was not available were excluded. A further five children were excluded due to incomplete data in the finger pattern recognition task. Note that the present study focused on a subset of tasks for which data was collected as part of a larger study investigating different aspects of finger-related skills and finger use for early numerical development. For the present study, all children, for which data of all measures relevant for the current study were available, were considered.

Sensitivity analyses were performed using G*power (Faul et al., 2009). With a desired power of .9 and an alpha level of .05, a sample of 101 children was sufficient to detect a small to medium effect of canonicity with Cohen's $g=.15$ for a sign test accounting for non-normally distributed data and of $d z=.29$ for a paired samples t-test (tested one-sided, 
FINGER PATTERNS IN EARLY CHILDHOOD

respectively). Moreover, to approximate sensitivity of a Friedman test with four factor levels (i.e., quantities one to four) and the dependent variable canonicity advantage (i.e., canonical non-canonical), a sensitivity analysis for a repeated measures ANOVA with adjusted sample size to account for reduced power in non-parametric designs was performed $(-15 \%$ of the sample for the corresponding parametric test resulting in $N=86$; Lehmann \& D'Abrera, 1998). With power set to .9 and an alpha level of .05, our study was sufficiently powered to detect a small to medium effect with $f=.13$.

Children were recruited from 17 kindergartens in the state of Baden-Württemberg in Germany. In the German education system, children usually spend three years in kindergarten (from around three to six years of age) before entering elementary school. Children were tested at the end of their second year of kindergarten and had no or limited prior exposure to formal learning contexts or activities as there is no curriculum for kindergartens in Germany.

Children participated only after written informed consent was obtained from parents or surrogates. Additionally, oral assent was obtained from children prior to testing. The study was approved by the local research ethics committee of the Leibniz-Institut für Wissensmedien, Tübingen.

\section{Tasks}

Finger pattern recognition. The computerised finger pattern recognition task consisted of pictures of finger patterns for quantities one to five that were presented canonically or non-canonically with one hand. Children were instructed to name the quantity depicted by a shown finger pattern as fast and accurately as possible. Finger patterns for quantity one to four were presented four times, twice per condition (i.e., canonical vs. noncanonical) and quantity. The finger pattern for quantity five was presented twice and not included in any of the analyses because it is not possible to present five non-canonically with 
FINGER PATTERNS IN EARLY CHILDHOOD

one hand. Thus, in total the task consisted of 18 items. The order of stimuli was pseudorandomised but fixed across participants.

The task started with a practice trial (finger pattern of quantity five). The instruction was repeated in case a child did not name a quantity or in case a child counted the fingers verbally and/or by pointing to each of the fingers. Children were not explicitly discouraged from using counting strategies. In case the child named a quantity incorrectly in the practice trial, the experimenter named the quantity correctly and repeated the instruction. No formative feedback was given for experimental trials.

To measure reaction times (RT; stimulus onset till button press), the experimenter pressed the spacebar as soon as the child uttered their answer for the quantity of fingers on the screen (see Schleifer \& Landerl (2010) for an evaluation of the appropriateness of such an approach). In addition to the correctness of the answer given by the child, the experimenter recorded whether the child showed any open counting strategies (e.g., verbally counting, pointing on the screen, nodding their head) and whether reaction times were corrupted (e.g., due to short-term inattention of the child caused by external distractors).

Three different dependent variables were considered for the analyses of the finger pattern recognition task: 1) number of correctly named finger patterns, 2) number of correctly recognised finger patterns (i.e., excluding trials in which the child counted openly), and 3) RTs for correctly recognised finger patterns (please refer to the results section for further details on data pre-processing of the RT data).

Verbal Counting. This task consisted of a total of 13 items adapted from the items used in subtest 1 of the standardised maths test TEDI-MATH (Kaufmann et al., 2009). First, children were asked to count as far as possible and received one point for correctly counting up to ten, two points for correctly counting to 20 , three points for correctly counting to 30 , and four points for correctly counting up to at least 31 . Subsequently, children were asked to 
FINGER PATTERNS IN EARLY CHILDHOOD

count until number $\mathrm{X}$ (three items, one point for naming the correct sequence each), to count onwards from a number other than one (two items, one point each), and to count from number X to Y (two items, also one point each). Children were further asked to name the successor and the predecessor of a number (two items each, one point per item). Finally, children were asked to count backwards from the number five. Here, children received one point in case at least two consecutive numbers were counted backwards, or two points in case they correctly counted backwards from five to one. Across all items, children could reach a maximum score of 17 points.

Quantity-number linkage. A sum score was computed across tasks tapping into quantity-number linkage, comprising the tasks quantity-number mapping, Give-N, and verbal number comparison. The maximum score across these tasks was 19.

Quantity-number mapping task (cf. MBK-0, Krajewski, 2018). Children were presented with a number band showing numbers 1 to 10 in Arabic digital notation. First, the experimenter explained the number band by naming all the numbers verbally and pointing to each of the Arabic digits while saying the corresponding number word. Children were then shown five pictures presenting different numbers of children. The experimenter then pointed to and named one of the numbers on the number band ("This is number X") and asked the child whether they could find the picture with children that corresponds to the number X (three items). Children were awarded one point for each correctly mapped picture.

Subsequently, the experimenter pointed to one of the remaining two pictures with children and asked which number on the number band corresponded to this particular picture (two items). Children again received one point for each correctly mapped picture. In this task, children could reach a maximum score of five points.

Give- $N$ task (cf. Wynn, 1992). Children were asked to give a certain quantity of wooden toy pears to a soft toy called Finn (e.g., Can you give Finn two pears?). The 
FINGER PATTERNS IN EARLY CHILDHOOD

quantities one through six were requested. Children were first asked to give one pear and then they were asked to give three pears. For all following trials a titration method (cf. Sarnecka \& Carey, 2008) was used: In case the correct quantity of pears was given, the subsequent quantity of pears requested increased by one and, vice versa, in case an incorrect quantity of pears was given, the subsequent quantity of pears requested was decreased by one. Aiming at identifying the knower-level of a child, this procedure continued until two criteria were met: 1) twice as many correct than erroneous responses were recorded for a certain quantity $\mathrm{N}$ and 2) none or twice as many erroneous than correct responses were recorded for the respective quantity $\mathrm{N}+1$ (e.g., in case one erroneous and two correct responses were recorded for $\mathrm{N}=4$ and two erroneous responses were recorded for $\mathrm{N}=5$ the task was terminated). For children that did not commit any error until $\mathrm{N}=6$, the task was terminated once the correct quantity of pears was given for $\mathrm{N}=6$.

The dependent variable for this task was the knower-level (i.e., corresponding to prenumber, one-/two-/three-/four-/five-, and six-knowers, respectively; cf. Le Corre, 2014; Le Corre et al., 2006; Wynn, 1992). Our prespecified termination criteria to determine the respective knower level did prove applicable for most but not all children. Some children appeared to currently transit from one knower-level to the next which resulted in a fairly high number of trials without meeting both criteria fully (e.g., jumping back and forth between two quantities because a child gave the correct quantity for each quantity in most trials but not always). Thus, for some children the experimenter terminated the task before both criteria were met $(\mathrm{N}=5$ children). To account for this, we assigned a knower level of $\mathrm{N}$ in case more than $50 \%$ of trials for $\mathrm{N}$ were correct and less than $50 \%$ of trials were correct for $\mathrm{N}+1$.

Moreover, we assigned a knower-level of N.5 (e.g., 3.5) in case more than $50 \%$ of trials for N were correct and exactly $50 \%$ of trials were correct for $\mathrm{N}+1$. Because for $\mathrm{N}=6$ the $\mathrm{N}+1$ criterion does not apply (e.g., we did not test quantities beyond six) children were considered 
FINGER PATTERNS IN EARLY CHILDHOOD

six-knowers in case they did not commit any error until $\mathrm{N}=6$ or in case they committed one error on the way up to quantity six which was checked and corrected immediately following the rules of the titration method.

Verbal number comparison. This task consisted of eight items. Children were asked to indicate whether a verbally presented number (i.e., one to four and six to nine) was less than five or more than five. Children received one point for each correct decision resulting in a maximum score of eight points for this task.

Non-symbolic Number Comparison. Children were briefly (i.e., one second) shown pictures with a number of objects on the left side and a different number of objects on the right side. For each of the three items, children were asked to point to the side where they had seen the larger number of objects. Children received one point for pointing to the correct side. Subsequently, the experimenter presented the picture again and asked children to check their answers. Children were allowed to count the objects in case they wanted to. In case they pointed to the correct side, children received an additional point per item. Children received an additional point in case they provided a correct explanation of why they pointed to the correct side (e.g., there are $\mathrm{X}$ objects on this side and $\mathrm{Y}$ objects on the other side, $\mathrm{X}$ is more than $\mathrm{Y}$, therefore, there are more objects on this side). In this task, children could reach a maximum score of nine points.

Additional materials used in each of the tasks can be accessed through the Open Science Framework project page (https://osf.io/t2bxc/).

\section{Procedure}

Children were tested individually by trained experimenters in their respective kindergarten. Whenever possible, children were assessed in a separate, quiet room to avoid distractions. All tasks (including those that are part of the larger study but were not 
FINGER PATTERNS IN EARLY CHILDHOOD

considered in the present study) were administered in two sessions. The duration of each session was approximately 20-30 minutes depending on the skills and needs of the child (e.g., need for a break). Time between testing sessions was at least one day but was kept to a minimum (i.e., for most children both sessions were completed within less than two weeks). The order of tasks was fixed across participants. The pattern recognition task was administered in session 1; whereas all other tasks used in the present study were administered in session 2 (in the order: verbal counting, non-symbolic number comparison, verbal number comparison, quantity-number mapping task, Give-N). At the end of each session, children received a small present (e.g., a sticker of their choice or a balloon).

To ensure a child-friendly and engaging environment, the study was embedded in a story about the alien Finn (see also Barrocas et al. (2019) for more details on the story and character Finn implemented in a game-based app). Children learned that, unfortunately, Finn does not have arms and fingers which makes it hard for him to learn about numbers. Therefore, children were encouraged to show Finn what they already know about fingers and numbers to help Finn learn more. A soft toy of the character Finn accompanied testing sessions and all tasks were embedded in a book explaining Finn's story. Finn was greatly appreciated by children and warmly welcomed on each testing day.

\section{Results}

The results section is split in two parts. The first part focuses on the analyses of the effect of canonicity in the finger pattern recognition task with respect to i) the number of correctly named finger patterns (i.e., either counted or recognised), ii) the number of correctly recognised finger patterns (i.e., excluding all trials for which children showed open counting strategies), and iii) RTs for correctly recognised finger patterns. To account for non-normally 
FINGER PATTERNS IN EARLY CHILDHOOD

distributed data and a high number of ties for accuracy data (i.e., instances in which the difference between conditions equals zero), exact sign tests were used to compare performance in the canonical and the non-canonical condition within participants and Friedman tests were used to investigate differences in the canonicity advantage for different quantities (i.e., quantities one to four). For RT analyses, paired samples t-tests were used.

The second part of the results section focuses on the association of finger pattern recognition performance with other numerical skills (i.e., counting, quantity-number linkage, and non-symbolic number comparison).

\section{Canonicity advantage}

Correctly named finger patterns. In a first step, we evaluated the number of correctly named finger patterns (i.e., either counted or recognised). Finger patterns showing quantity five were not included in any of the analyses because the finger pattern for quantity five is always canonical when presented with one hand. Overall children did well in this task as overall $91.9 \%$ of finger patterns [canonical: $92.3 .0 \%$; non-canonical: $91.6 \%$ ] were correctly named. On average children named $M=14.6$ out of 16 patterns correctly $[S D=2.7$; range 1 16] with nearly identical mean accuracy for canonical $[M=7.3, S D=1.4]$ and non-canonical finger patterns $[M=7.3, S D=1.5]$.

In line with findings reported for the youngest group of children (aged four to five) in the study by Lafay and colleagues (2013), an exact sign test showed no significant effect of canonicity $[z=-0.50, p=.617]$ with 65 ties, 20 children performing better in the canonical condition and 16 children performing better in the non-canonical condition. Thus, there was no significant difference in accuracy between the canonical and the non-canonical condition when there was no further distinction between counted and recognised trials. 
FINGER PATTERNS IN EARLY CHILDHOOD

To investigate whether the effect of canonicity varied across quantities one to four, a Friedman test was run with the factor quantity (one vs. two vs. three vs. four) and the dependent variable canonicity advantage for correctly named finger patterns (canonical - noncanonical). No significant effect of quantity was found $\left[\chi^{2}(3)=0.17, p=.982\right]$ providing no evidence for a difference in the canonicity advantage for different quantities. Indeed, separate sign tests for each of the four quantities were all non-significant [all $p>.754$ ]

Accuracy of correctly recognised finger patterns. In a second step, we analysed the number of correctly recognised finger patterns (i.e., excluding all trials for which children showed open counting strategies). A to be expected drop in performance was observed showing that, overall, $60.2 \%$ of finger patterns were correctly recognised [canonical: $62.8 \%$; non-canonical: $57.6 \%$ ]. On average children recognised $M=9.5$ out of 16 finger patterns correctly $[S D=6.3$; range $0-16$; canonical: $M=5.0, S D=3.2$; non-canonical: $M=4.6, S D=$ 3.2]. An exact sign test on the number of correctly recognised finger patterns indicated a significant effect of canonicity $[z=-2.63, p=.009]$ with 54 ties, 33 children performing better in the canonical condition and 14 children performing better in the non-canonical condition. Thus, although we observed a significant canonicity effect on the group level, considerable variability was observed on the individual level.

Regarding differences in the size of the canonicity advantage for the quantities one to four when considering the number of correctly recognised finger patterns, a further Friedman test was run with the factor quantity (one vs. two vs. three vs. four) and the dependent variable canonicity advantage for correctly named finger patterns (canonical - noncanonical). Results showed a significant effect of quantity $\left[\chi^{2}(3)=22.29, p<.001\right]$. Results of four different follow up sign tests for each quantity showed a significant canonicity advantage for the quantities three and four [three: $p<.001$; ties: $N=77, \mathrm{C}>\mathrm{NC}: N=21, \mathrm{NC}$ 
FINGER PATTERNS IN EARLY CHILDHOOD

$>\mathrm{C}: N=3$; four: $p<.001$; ties: $N=72, \mathrm{C}>\mathrm{NC}: N=24, \mathrm{NC}>\mathrm{C}: N=5]$ but not for quantities one and two $[p>.999$ and $p=.648$, respectively]. As illustrated in Figure 1, the observed overall canonicity advantage seems to be largely driven by quantities three and four and by a subset of children performing better in canonical compared to non-canonical finger pattern recognition.

Reaction times for correctly recognised finger patterns. Thirdly, we analysed reaction times for correctly recognised finger patterns. For RT analyses, all trials for which children showed open counting strategies as well as trials with corrupted RTs as documented by the experimenter were excluded. Moreover, for each quantity (i.e., one to four) and condition (canonical vs. non-canonical) the respective faster RT out of the two repetitions was considered only. Subsequently, RTs slower than $2 S D$ above the overall mean RT for each quantity, respectively, were excluded from the analyses $(N=13$ trials).

Finally, to ensure comparability between the canonical and the non-canonical condition and to avoid biassing number magnitude effects due to the imbalanced number of data points per condition, we adopted a quantity matching approach for estimating RTs. In particular, RT for a certain quantity (i.e., one to four) was only included in case a valid RT was available for both the canonical and the non-canonical condition (e.g., if there was a valid RT for canonical finger patterns two, three and four, but only for non-canonical finger patterns two and three, mean RTs were calculated and included only for quantities two and three in both conditions). Some children did not recognise any or very few finger patterns (e.g., because they counted). For these children our quantity matching approach resulted in no matched pairs. Thus, these children were not included in the analyses leading to a reduced sample of $N=67$ for RT analyses. 
FINGER PATTERNS IN EARLY CHILDHOOD

In line with results for the number of correctly recognised finger patterns, a pairedsamples t-test on RTs for correctly recognised finger patterns indicated that - averaged across quantities one to four - children responded significantly faster to canonical finger patterns $[M$ $=2284 \mathrm{~ms}, S D=471 \mathrm{~ms}]$ than to non-canonical finger patterns $[M=2086 \mathrm{~ms}, S D=428 \mathrm{~ms}$; $t(66)=3.61, p<.001$, Cohen's $d=.44]$.

Our quantity matching approach resulted in an unequal number of data points (children) for quantities one to four and, thus, does not represent a full within-subject factorial design. Therefore, it was not feasible to run a factorial analysis (e.g., ANOVA) to investigate differences in the size of the canonicity advantage across quantities; instead, we ran separate t-tests for each of the four quantities to investigate whether there was a significant effect of canonicity for each of the quantities.

Mirroring results reported for the number of correctly recognised finger patterns, separate t-tests for each quantity showed that canonical finger patterns were recognised significantly faster for quantities three and four [three: $t(54)=4.40, p<.001$, Cohen's $d=.59$, canonical: $M=2030 \mathrm{~ms}, S D=585 \mathrm{~ms}$, non-canonical: $M=2479 \mathrm{~ms}, S D=759 \mathrm{~ms}$; four: $t(42)=$ 3.74, $p<.001$, Cohen's $d=.57$, canonical: $M=2434 \mathrm{~ms}, S D=617 \mathrm{~ms}$, non-canonical: $M=$ $2977 \mathrm{~ms}, S D=1038 \mathrm{~ms}$ ]. However, no significant difference was found for quantities one and two $[p=.979$ and $p=.681$, respectively; cf. Figure 1]. 
FINGER PATTERNS IN EARLY CHILDHOOD

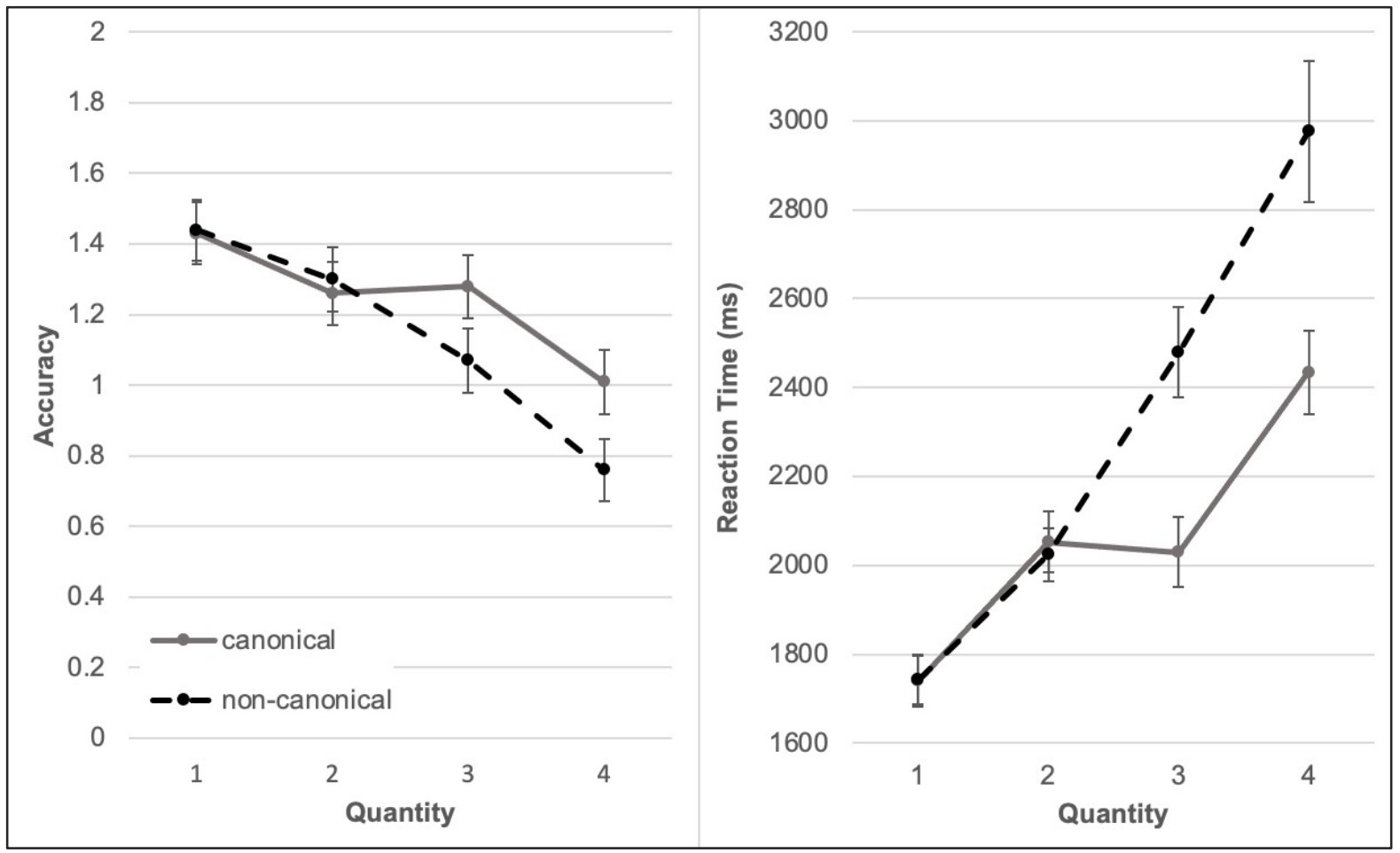

Figure 1. Canonicity advantage for finger patterns of quantities one to four.

Canonicity advantage presented separately for the number of correctly recognised finger patterns (left panel) and reaction times of correctly recognised finger patterns (right panel). Error bars represent standard errors of the means.

\section{Associations of finger pattern recognition and numerical skills}

Both canonical and non-canonical finger pattern recognition performance showed moderate to strong associations with both counting skills and quantity-number linkage, but non-significant or only small associations with non-symbolic number comparison (see Table 1). Importantly, the correlations of finger pattern recognition performance (i.e., correctly named as well as correctly recognised finger patterns) with both counting skills and quantitynumber linkage were comparable in size for canonical and non-canonical finger patterns.

A to be expected drop in the size of correlations between finger pattern recognition and numerical skills was observed when controlling for verbal counting skills. Indeed, with only two exceptions, correlations were no longer significant when controlling for verbal 
FINGER PATTERNS IN EARLY CHILDHOOD

counting skills and after correction for multiple comparisons for the 30 correlations of interest (i.e., correlations of the three dependent variables for canonical and non-canonical finger pattern recognition performance with the five numerical tasks) using the procedure suggested by Benjamini and Hochberg (1995). In particular, accuracy in canonical finger pattern recognition was positively correlated with performance in the verbal number comparison task and reaction times in canonical finger pattern recognition was negatively correlated with the combined quantity-number linkage score. Thus, both correlations that remained significant after controlling for counting skills were found for canonical but not non-canonical finger pattern performance and for finger pattern recognition but not finger pattern naming performance (i.e., considering only trials without open counting strategies). To investigate whether verbal number comparison and quantity-number linkage were differentially related to canonical vs. non canonical finger pattern recognition, respectively, we used the method suggested by Eid et al. (2011) for comparing two dependent correlations. Results indicated no significant difference between the correlation of verbal number comparison and the number of recognised canonical vs. non-canonical finger patterns $[p=$ .152]. Contrarily, a significantly larger correlation was found between quantity-number linkage and reaction times for the recognition of canonical $[r=-.315]$ as compared to noncanonical finger patterns $[r=-.091 ; z=-2.05, p=.02]$. 


\section{FINGER PATTERNS IN EARLY CHILDHOOD}

Table 1. Spearman correlation coefficients, partial correlations, and descriptive statistics.

\begin{tabular}{|c|c|c|c|c|c|c|c|c|c|c|c|c|}
\hline & 1 & 2 & 2.1 & 2.2 & 2.3 & 3 & 4.1 & 4.2 & 5.1 & 5.2 & 6.1 & 6.2 \\
\hline (1) Verbal counting & & $.761 * *$ & $.651 * *$ & $.586 * *$ & $.677 * *$ & $.356^{* *}$ & $.495 * *$ & $.448 * *$ & $.481 * *$ & $.532 * *$ & $-.449 * *$ & $-.472 * *$ \\
\hline (2) Quantity-number linkage & & & $.864^{* *}$ & $.706^{* *}$ & $.834 * *$ & $.399 * *$ & $.454 * *$ & $.442 * *$ & $.469 * *$ & $.458 * *$ & $-.508 * *$ & $-.371 *$ \\
\hline (2.1) Quantity-number mapping & & $.749 * *$ & & $.598 * *$ & $.515 * *$ & $.318^{* *}$ & $.361 * *$ & $.344 * *$ & $.358 * *$ & $.367 * *$ & $-.399 * *$ & $-.267^{\circ}$ \\
\hline (2.2) Give-N & & $.496 * *$ & $.352 * *$ & & $.572 * *$ & $.367 * *$ & $.351 * *$ & $.401 * *$ & $.390 * *$ & $.376 * *$ & $-.348 *$ & $-.343 *$ \\
\hline (2.3) Verbal number comparison & & $.668 * *$ & .133 & $.295 *$ & & $.364 * *$ & $.452 * *$ & $.400 * *$ & $.458 * *$ & $.442 * *$ & $-.315^{*}$ & $-.369 *$ \\
\hline (3) Non-symbolic number comparison & & $.211^{\circ}$ & .121 & $.209^{\circ}$ & .179 & & .129 & .150 & $.264^{\circ}$ & $.238^{\circ}$ & -.117 & -.129 \\
\hline \multicolumn{13}{|l|}{ Finger patterns: correctly named ACC } \\
\hline (4.1) Canonical & & .138 & .059 & .086 & .183 & -.059 & & $.579 * *$ & $.596 * *$ & $.520 * *$ & $-.379 *$ & -.114 \\
\hline (4.2) Non-Canonical & & .175 & .078 & .191 & .148 & -.012 & $.459 * *$ & & $.279^{*}$ & $.390 * *$ & $-.314^{\circ}$ & $-.331^{\circ}$ \\
\hline \multicolumn{13}{|l|}{ Finger patterns: correctly recognised ACC } \\
\hline (5.1) Canonical & & .181 & .068 & .152 & $.206^{\circ}$ & .113 & & & & $.870 * *$ & $-.443 * *$ & -.217 \\
\hline (5.2) Non-Canonical & & .097 & .032 & .094 & .132 & .061 & & & $.828 * *$ & & $-.459 * *$ & $-.432 * *$ \\
\hline \multicolumn{13}{|l|}{ Finger patterns: correctly recognised RT } \\
\hline (6.1) Canonical & & $-.315^{\circ}$ & -.192 & -.180 & -.111 & .035 & & & & & & $.506 * *$ \\
\hline (6.2) Non-Canonical & & -.091 & -.008 & -.163 & -.167 & -.029 & & & & & $.373 *$ & \\
\hline Mean & 8.11 & 14.17 & 2.66 & 5.30 & 6.21 & 6.63 & & & & & & \\
\hline Standard deviation & 4.29 & 4.37 & 1.87 & 1.37 & 2.06 & 1.58 & & & & & & \\
\hline Range & $1-17$ & $3-19$ & $0-5$ & $1-6$ & $0-8$ & $0-9$ & & & & & & \\
\hline Maximum & 17 & 19 & 5 & 6 & 8 & 9 & & & & & & \\
\hline
\end{tabular}

Note. $N=67$ for Finger pattern recognition RT, $N=101$ for all other tasks. Spearman correlations are presented above the diagonal, Spearman partial correlations controlling for verbal counting skills are presented below the diagonal. ACC = accuracy; RT = reaction time; correctly named $=$ either counted or recognised finger patterns; correctly recognised $=$ all trials for which children showed no open counting strategies; $* *: p \leq$

$.001, *: \mathrm{p} \leq .005,{ }^{\mathrm{o}}: \mathrm{p} \leq .05$ 
FINGER PATTERNS IN EARLY CHILDHOOD

\section{Discussion}

The present study set out to deepen our understanding of the developmental onset of finger pattern recognition - and with this the development of an embodied, finger-based representation of number magnitude - as well as its relation to concurrent numerical skills in young children around the age of four years. Children were asked to do a finger pattern recognition task requiring them to name the quantity depicyed as canonical or non-canonical finger patterns as quickly as possible. Additionally, children's counting, quantity-number linkage (i.e., number mapping task, Give-N, verbal number comparison) as well as nonsymbolic number comparison skills were assessed. Generalising findings from previous studies in older children, results of the present study showed a canonicity advantage already for children around the age of four years when considering only those trials that were solved without open counting strategies. Crucially, the observed canonicity advantage on the group level seemed to be driven by a subset of children, who performed better in canonical than in non-canonical finger pattern recognition. This corroborates the idea of substantial interindividual differences in the emergence of finger-based representations of number magnitude in young children. Moreover, medium to large correlations were observed for both canonical and non-canonical finger pattern recognition with verbal counting skills and tasks tapping into quantity-number linkage. However, relations with quantity-number linkage were heavily driven by verbal counting skills. In fact, correlations that remained significant when controlling for verbal counting skills were only found for canonical but not non-canonical finger pattern recognition performance (i.e., considering only trials without open counting strategies). Taken together the present results support the idea of a comparably early but also developmentally scattered emergence of the canonicity advantage in finger-pattern 
FINGER PATTERNS IN EARLY CHILDHOOD

recognition for children as young as four years of age and its potential significance for tasks tapping into quantity-number linkage.

Previous research indicates that adults seem to automatically activate iconic, fingerbased representations when processing numerical information through canonical finger patterns (e.g., Di Luca \& Pesenti, 2008). Studies in adults are highly informative with respect to the precise working mechanisms underlying numerical processing in general and iconic, finger-based representations, in particular. However, adult numerical performance - as the product of the mastery of major steps throughout numerical development - provides little insight about the onset and emergence of such iconic, finger-based representations in children. The present study contributes to our understanding of the emergence of iconic, finger-based representations of number magnitude by zooming in into an earlier developmental time window than most studies have focused on so far. Previous research indicated that children aged five years and older showed an advantage in recognising canonical as compared to non-canonical finger patterns (Kreilinger et al., 2021; Lafay et al., 2013; Marlair et al., 2021; Noël, 2005) whereas the evidence for even younger children is more limited and less clear. Specifically, Lafay and colleagues (2013) observed no evidence for a processing advantage of canonical as compared to non-canonical finger patterns in a finger pattern recognition task in children aged four to five years. Conducting a comparable analysis considering all trials (counted and recognised), the present study also failed to find a significant canonicity advantage. However, considering only those trials in which the quantities were indeed recognised and not solved by open counting strategies, results of the present study indicated a significant canonicity advantage in finger pattern recognition for children around the age of four years. This canonicity effect was consistently found in both accuracy and reaction time data. Importantly, however, the canonicity advantage was not found for all quantities used and not for all children: it was present for quantities three and 
FINGER PATTERNS IN EARLY CHILDHOOD

four and, considering the individual level, it was found only for around a fourth to a third of children.

Regarding the particular quantity range used, the present study corroborates but also extends previous findings showing that the canonicity advantage depends on the quantity range and age group under investigation (Kreilinger et al., 2021; Noël, 2005; Marlair et al., 2021). In particular, while previous studies in older children showed a canonicity advantage for quantities larger than four, we were able to demonstrate a canonicity advantage for children aged around four years for quantities three and four. Considering both past and present findings, the available evidence may suggest that especially for the recognition of small quantities (i.e., one to four) in older children, a lack or decreased effect size of a canonicity advantage may also be explained by subitising skills. This means that small sets of objects - irrespective of whether they are depicted canonically or non-canonically - have been shown to be recognised quickly and effortlessly without counting ( e.g., Kaufmann et al., 1949). While the lack/decreased effect size of a canonicity advantage for smaller numbers in older children does not necessarily question the existence of canonical, finger-based representations of number, canonical finger-based representations may, however, provide a comparably minor contribution for facilitating the recognition process of small sets in older children. Importantly, however, observing a processing advantage for canonical finger patterns within the subitising range (i.e., quantities one to four) in young children is in line with the idea of a facilitating effect of (emerging) finger-based representations of number magnitude over and above additional subitising skills.

Crucially, however, our results further highlight the fact that there seem to be substantial inter-individual differences in the onset of a canonicity advantage which may influence the age at which the emergence of the canonicity advantage in children may be observed on the group level. The fact that (some) children aged about four years already 


\section{FINGER PATTERNS IN EARLY CHILDHOOD}

showed a canonicity advantage may suggest that children around this age start to develop iconic, finger-based representations that eventually allow them to directly access number magnitude and potentially facilitates the linkage of non-symbolic quantities with symbolic representations of number (i.e., number words or Arabic digits; Fayol \& Seron, 2005, see also Butterworth, 1999). And indeed, highlighting the potential significance of iconic, fingerbased representations of number magnitude for numerical development, our study provides initial, though somewhat inconsistent evidence suggesting that canonical finger pattern recognition - not non-canonical - seemed specifically associated with better quantity-number linkage skills after controlling for verbal counting skills.

However, the question arises whether an observed canonicity advantage in young children is indeed already driven by the same working mechanisms that have been observed in adults - that is direct access to number magnitude through iconic, finger-based representations (e.g., Di Luca \& Pesenti, 2008). In particular, even when considering only those trials for which no open counting strategies could be observed, mean reaction times for both canonical and non-canonical finger patterns were still longer than two seconds.

Moreover, correlations between canonical (and non-canonical) finger pattern recognition and tasks tapping into quantity-number linkage dropped considerably and - with two exceptions were no longer significant after controlling for verbal counting skills.

Thus, although we accounted for open counting strategies in our analyses, the observed correlations still seemed to be heavily driven by verbal counting skills. This finding may suggest that the observed difference in canonical vs. non-canonical finger pattern recognition may be a by-product of individual differences in fast (verbal) counting skills rather than or in addition to (fully automatic) access to number magnitude through emerging but not fully established iconic, finger-based representations. Whether children already automatically and directly access number magnitude information similar to adults (cf. Di 
FINGER PATTERNS IN EARLY CHILDHOOD

Luca \& Pesenti, 2008) may thus be debated and awaits further systematic experimental work across age groups.

Crucially though, even if children used fast counting skills rather than direct recognition through iconic, finger-based representations to access number magnitude information, the canonicity of finger patterns still mattered and facilitated task execution. Thus, canonical finger patterns may have allowed children to speed up and systematise processing through their familiar iconic format. Through repeated exposure and practice, the proportion of children relying on counting strategies is expected to drop with further development because counting strategies may eventually be replaced by direct, automatic access to number magnitude through iconic, finger-based representations of number magnitude.

As such, iconic, embodied finger-based representations of number magnitude may indeed represent the "missing link" (Fayol \& Seron, 2005, see also Butterworth, 1999) for relating symbolic number representations (i.e., number words and Arabic digits) with nonsymbolic quantities. This idea is also in line with results by Gibson et al. (2019) who found that gesture-speech mismatches (i.e., tagging a quantity correctly with fingers but incorrectly with a number word) were indicative of a readiness to learn new numbers (i.e., mapping of non-symbolic quantities to number words). Although they did not look into canonicity specifically, this finding fits nicely with the idea of finger patterns constituting a transitional link between non-symbolic and symbolic representations of numbers. Future research endeavours may tackle this coevolution more directly by tracing the developmental footprint of both (verbal) counting skills, finger-based representations, and quantity-number linkage skills in longitudinal, potentially micro-genetic study designs to investigate the sequential or parallel developmental trajectories.

\section{Conclusion}


FINGER PATTERNS IN EARLY CHILDHOOD

Generalising findings from previous studies in older children, results of the present study showed a canonicity advantage in finger pattern recognition for children as young as four years of age. However, the canonicity advantage was not found for all quantities used and not for all children suggesting a comparably early but nevertheless developmentally scattered emergence of a canonicity advantage in finger-pattern recognition which was linked to counting skills as well as skills tapping into quantity-number linkage. Thus, canonical finger patterns seem to facilitate the mapping of finger patterns to number magnitude for (some) children aged around four years albeit potentially not (yet) through a fully established iconic, finger-based representation of number magnitude.

\section{Author Contribution}

All authors contributed to the study conception and design. Material preparation, project administration, data collection, curation, and analysis were performed by JB and RB. The first draft of the manuscript was written by JB and all authors commented on previous versions of the manuscript. SR and KM acquired the funding for the study. All authors read and approved the final manuscript.

\section{Competing Interest Statement}

All authors certify that they have no affiliations with or involvement in any organisation or entity with any financial interest or non-financial interest in the subject matter or materials discussed in this manuscript. 
FINGER PATTERNS IN EARLY CHILDHOOD

\section{Data, material, and analysis script availability statement}

The datasets generated during and/or analysed in the current study are not publicly available, as the sharing of data publicly was not covered by the informed consent. The data is available from the corresponding author upon request.

Materials used in this study as well as the analysis syntax can be accessed through the Open Science Framework project page (https://osf.io/t2bxc/).

\section{Acknowledgements}

This research was funded by the German Research foundation [grant number: 406023305]. We thank Alexandra Vetter, Carla Schroepel, Dennis Hafner, Hannah Sindram, Johannes Erdmann, Manuel Koepper, Michelle Schimmock, Nicole Antes, Stefanie Arnold, Svenja Maas, and Vanessa Bartelt for their help in data collection and curation. We especially also thank all participating kindergartens, parents, and children for supporting our research before and during a global pandemic.

\section{References}

Andres, M., Di Luca, S., \& Pesenti, M. (2008). Finger counting: The missing tool?. Behavioral and Brain Sciences, 31(6), 642-643. https://doi.org/10.1017/S0140525X08005578

Barrocas, R., Roesch, S., Dresen, V., Moeller, K., \& Pixner, S. (2020). Embodied numerical representations and their association with multi-digit arithmetic performance. Cognitive Processing, 21(1), 95-103. https://doi.org/10.1007/s10339-019-00940-z

Barrocas, R., Ninaus, M., Tsarava, K., Gawrilow, C., Lachmair, M., Roesch, S., \& Moeller, K. (2019). Digits grasp digits: Numbers on your fingertips. Proceedings of the 11th International Conference on Education and New Learning Technologies 
FINGER PATTERNS IN EARLY CHILDHOOD

(EDULEARN19) (pp. 7165-7174). Valencia: IATED Academy.

https://doi.org/10.21125/edulearn.2019.1714

Barsalou LW (2008) Grounded cognition. Annual Review of Psychology, 59, 617-645. https://doi.org/10.1146/annurev.psych.59.103006.093639

Beller, S., \& Bender, A. (2011). Explicating numerical information: when and how fingers support (or hinder) number comprehension and handling. Frontiers in Psychology, 2:214. https://doi.org/10.3389/fpsyg.2011.00214

Benjamini, Y., \& Hochberg, Y. (1995). Controlling the false discovery rate: a practical and powerful approach to multiple testing. Journal of the Royal Statistical Society: Series B (Methodological), 57(1), 289-300. https://doi.org/10.1111/j.2517-6161.1995.tb02031.x

Butterworth, B. (1999). The mathematical brain. London: Macmillan.

Cornu, V., Schiltz, C., Martin, R., \& Hornung, C. (2018). Visuo-spatial abilities are key for young children's verbal number skills. Journal of Experimental Child Psychology, 166, 604-620. https://doi.org/10.1016/j.jecp.2017.09.006

Crollen, V., \& Noël, M. P. (2015). The role of fingers in the development of counting and arithmetic skills. Acta Psychologica, 156, 37-44.

https://doi.org/10.1016/j.actpsy.2015.01.007

Crollen, V., Seron, X., \& Noël, M. P. (2011). Is finger-counting necessary for the development of arithmetic abilities? Frontiers in Psychology, 2:242. https://doi.org/10.3389/fpsyg.2011.00242

Di Luca, S., \& Pesenti, M. (2008). Masked priming effect with canonical finger numeral configurations. Experimental Brain Research, 185(1), 27-39. https://doi.org/10.1007/s00221-007-1132-8

Di Luca, S., \& Pesenti, M. (2011) Finger numeral representations: more than just another symbolic code. Frontiers in Psychology, 2:272. https ://doi.org/10.3389/fpsyg .2011 .00272 
FINGER PATTERNS IN EARLY CHILDHOOD

Di Luca, S., Lefèvre, N., \& Pesenti, M. (2010). Place and summation coding for canonical and non-canonical finger numeral representations. Cognition, 117(1), 95-100. https://doi.org/10.1016/j.cognition.2010.06.008

Eid, M., Gollwitzer, M., \& Schmitt, M. (2011). Statistik und Forschungsmethoden Lehrbuch. Weinheim: Beltz.

Faul, F., Erdfelder, E., Buchner, A., \& Lang, A. G. (2009). Statistical power analyses using $\mathrm{G}^{*}$ Power 3.1: Tests for correlation and regression analyses. Behavior Research Methods, 41(4), 1149-1160. https://doi.org/10.3758/BRM.41.4.1149

Fayol, M., \& Seron, X. (2005). About numerical representations: Insights from neuropsychological, experimental, and developmental studies. In J. I. D. Campbell (Ed.), Handbook of mathematical cognition (pp. 3-22). New York: Psychology Press.

Gibson, D. J., Gunderson, E. A., Spaepen, E., Levine, S. C., \& Goldin-Meadow, S. (2019). Number gestures predict learning of number words. Developmental Science, 22: e12791. https://doi.org/10.1111/desc.12791

Gunderson, E. A., Spaepen, E., Gibson, D., Goldin-Meadow, S., \& Levine, S. C. (2015). Gesture as a window onto children's number knowledge. Cognition, 144, 14-28. https://doi.org/10.1016/j.cognition.2015.07.008

Hornung, C., Martin, R., \& Fayol, M. (2017). General and specific contributions of RAN to reading and arithmetic fluency in first graders: A longitudinal latent variable approach. Frontiers in Psychology, 8:1746. https://doi.org/10.3389/fpsyg.2017.01746

Kaufman, E., Lord, M., Reese, T., \& Volkmann, J. (1949). The discrimination of visual number. The American Journal of Psychology, 62(4), 498-525. https://doi.org/10.2307/1418556

Kaufmann, L., Nuerk, H. C., Graf, M., Krinzinger, H., Delazer, M., \& Willmes, K. (2009). TEDI-MATH: Test zur Erfassung numerisch-rechnerischer Fertigkeiten vom Kindergarten bis zur 3. Klasse. Bern: Hans Huber.

Krajewski, K. (2018). MBK 0: Test mathematischer Basiskompetenzen im Kindergartenalter. Hogrefe. 
FINGER PATTERNS IN EARLY CHILDHOOD

Kreilinger, I. L., Roesch, S., Moeller, K., \& Pixner, S. (2021). Mastery of structured quantities like finger or dice patterns predict arithmetic performance. Cognitive Processing, 22(1), 93-104. https://doi.org/10.1007/s10339-020-00994-4

Lafay, A., Thevenot, C., Castel, C., \& Fayol, M. (2013). The role of fingers in number processing in young children. Frontiers in Psychology, 4:488. https://doi.org/10.3389/fpsyg.2013.00488

Lakoff, G., \& Núñez, R. (2000). Where mathematics comes from (Vol. 6). New York: Basic Books.

Le Corre, M. (2014). Children acquire the later-greater principle after the cardinal principle. British Journal of Developmental Psychology, 32(2), 163-177. https://doi.org/10.1111/bjdp.12029

Le Corre, M., Van de Walle, G., Brannon, E. M., \& Carey, S. (2006). Re-visiting the competence/performance debate in the acquisition of the counting principles. Cognitive Psychology, 52(2), 130-169. https://doi.org/10.1016/j.cogpsych.2005.07.002

Lehmann, E. L., \& D’Abrera, H. J. M. (1998). Nonparametrics: Statistical methods based on ranks (rev. ed.). Prentice-Hall.

Marlair, C., Lochy, A., Buyle, M., Schiltz, C., \& Crollen, V. (2021). Canonical representations of fingers and dots trigger an automatic activation of number semantics: an EEG study on 10-year-old children. Neuropsychologia, 157:107874. https://doi.org/10.1016/j.neuropsychologia.2021.107874

Moeller, K., Fischer, U., Link, T., Wasner, M., Huber, S., Cress, U., \& Nuerk, H. C. (2012). Learning and development of embodied numerosity. Cognitive Processing, 13(1), 271274. https://doi.org/10.1007/s10339-012-0457-9

Noël, M.-P. (2005). Finger gnosia: a predictor of numerical abilities in children? Child Neuropsychology, 11(5), 413-430. https://doi.org/10.1080/09297040590951550

Sarnecka, B. W., \& Carey, S. (2008). How counting represents number: what children must learn and when they learn it. Cognition, 108(3), 662-74. https://doi.org/10.1016/j.cognition.2008.05.007 
Schleifer, P., \& Landerl, K. (2011). Subitizing and counting in typical and atypical development. Developmental Science, 14(2), 280-291. https://doi.org/10.1111/j.14677687.2010.00976.x

Soylu, F., Rivera, B., Anchan, M., \& Shannon, N. (2019). ERP differences in processing canonical and noncanonical finger-numeral configurations. Neuroscience Letters, 705 , 74-79. https://doi.org/10.1016/j.neulet.2019.04.032

van den Berg, F. C., de Weerd, P., \& Jonkman, L. M. (2021). Electrophysiological evidence for internalized representations of canonical finger-number gestures and their facilitating effects on adults' math verification performance. Scientific Reports, 11(1), 1-15. https://doi.org/10.1038/s41598-021-91303-2

van Rinsveld, A., Hornung, C., \& Fayol, M. (2020). Finger Rapid Automatized Naming (RAN) predicts the development of numerical representations better than finger gnosis. Cognitive Development, 53:100842. https://doi.org/10.1016/j.cogdev.2019.100842

Wynn, K. (1992). Children's acquisition of the number words and the counting system. Cognitive Psychology, 24(2), 220-251. https://doi.org/10.1016/0010-0285(92)90008-P 